\title{
BRAID REPRESENTATIONS FROM UNITARY BRAIDED VECTOR SPACES
}

\author{
CÉSAR GALINDO AND ERIC C. ROWELL
}

\begin{abstract}
We investigate braid group representations associated with unitary braided vector spaces, focusing on a conjecture that such representations should have virtually abelian images in general and finite image provided the braiding has finite order. We verify this conjecture for the two infinite families of Gaussian and group-type braided vector spaces, as well as the generalization to quasibraided vector spaces of group-type.
\end{abstract}

\section{INTRODUCTION}

In this article we study certain unitary representations of braid group $\mathcal{B}_{n}$ generated by $\sigma_{1}, \ldots, \sigma_{n-1}$ satisfying:

(R1) $\sigma_{i} \sigma_{i+1} \sigma_{i}=\sigma_{i+1} \sigma_{i} \sigma_{i+1}$

(R2) $\sigma_{i} \sigma_{j}=\sigma_{j} \sigma_{i}$ for $|i-j|>1$.

Sequences of unitary representations of $\mathcal{B}_{n}$ are of central importance in the topological model for quantum computation, in which the computational power is intertwined with the sizes of the closed images of the unitary braid group action on the state spaces of $n$-punctured disks. In this context the images of the braid group generators $\sigma_{i}$ serve as quantum gates, and the goal is to determine when braiding alone is universal (i.e., any unitary operator can be efficiently approximated as the image of a braid, within a given error threshold). For universal braiding it is essentially sufficient to show that the closed images of the braid group $\mathcal{B}_{n}$ on each irreducible sector $V$ contains $S U(V)$, for $n$ large enough.

The unitary braid representations in this setting are described algebraically through unitary braided fusion categories. An object $X$ in such a category $\mathcal{C}$ gives rise, through the braiding operators $c_{X, X} \in \operatorname{End}_{\mathcal{C}}\left(X^{\otimes 2}\right)$, to unitary braid group representations $\rho_{X}^{n}$ on $\operatorname{End}_{\mathcal{e}}\left(X^{\otimes n}\right)$. Geometrically, this corresponds to disks with $n$-punctures, each labeled by the object $X$ (with boundary label varying over all simple objects).

C.G. would like to thank the hospitality of the Mathematics Departments at MIT and Texas A\&M where part of this work was carried out. C.G. was partially supported by Vicerrectoría de Investigaciones de la Universidad de los Andes. E.C.R. gratefully acknowledges the partial support of US NSF grant DMS-1108725 and the hospitality and support of BICMR, Peking University where part of this article was prepared. 
Recent articles devoted to determining images of unitary braid group representations include: [13, 25, 24, 30] while Jones [19] seems to have been the first to consider the problem of characterizing the images. In [13, 25] the cases where the braid group images are imprimitive, decomposable or finite are eliminated and then the remaining possibilities are analyzed in terms of the eigenvalues of the generators $\sigma_{i}$, using Lie theory.

Braid group representations emerge in quantum information in another context through the Yang-Baxter equation ([23]), as entanglement resources. Recall that $c \in \operatorname{Aut}\left(V^{\otimes 2}\right)$ satisfies the Yang-Baxter equation if

$$
\left(c \otimes I_{V}\right)\left(I_{V} \otimes c\right)\left(c \otimes I_{V}\right)=\left(I_{V} \otimes c\right)\left(c \otimes I_{V}\right)\left(I_{V} \otimes c\right) .
$$

In this case the pair $(V, c)$ will be called a braided vectors space (BVS). Given a BVS, $(V, c)$, we obtain braid group representations $\rho^{c}: \mathcal{B}_{n} \rightarrow G L\left(V^{\otimes n}\right)$ defined on generators by $\rho^{c}\left(\sigma_{i}\right)=I^{\otimes(i-1)} \otimes c \otimes I^{\otimes(n-i-1)}$.

A connection between braid group representations in these two quantum computational contexts was established and generalized in [28, 14] using the concept of localization. Roughly, a localization of the sequence of representations $\rho_{X}^{n}$ is a $\operatorname{BVS}(V, c)$ that faithfully encodes $\rho_{X}^{n}$, uniformly for all $n$. It is conjectured in loc. cit. that the sequence of representations $\rho_{X}^{n}$ are localizable if, and only if, $\operatorname{dim}(X)^{2} \in \mathbb{N}$. On the other hand, the property $F$ conjecture (see [27]) predicts that the braid group representations $\rho_{X}^{n}$ have finite image if, and only if, $\operatorname{dim}(X)^{2} \in \mathbb{N}$.

Motivated by the above we consider the problem of characterizing the images of the braid group representation associated with a unitary BVS. Recall that a virtually abelian group is a group with an abelian subgroup of finite index. We make the following:

Conjecture 1.1. Suppose $(V, c)$ is a unitary BVS.

(a) Then $\rho^{c}\left(\mathcal{B}_{n}\right)$ is a virtually abelian group for all $n$.

(b) If in addition $c$ has finite order then $\rho^{c}\left(\mathcal{B}_{n}\right)$ is finite.

The validity of this conjecture would imply, for example, that if $(V, c)$ is a unitary BVS, $\{c\}$ is never a universal gate set.

Unitary braid representations are abundant: for example Wenzl [32] showed that the braided fusion categories associated with quantum groups at roots of unity are usually unitary, and hence produce many examples. However, despite their ubiquity throughout mathematics and physics, there are remarkably few unitary BVSs in the literature. A classification of BVSs is only known for $\operatorname{dim}(V)=2$ [8, 18], for which Conjecture 1.1 follows from [12, 11]. In this article we focus on two infinite families: the so-called Gaussian BVSs (see Section 3 and [15]) and group-type BVSs (see Section 4). In particular, we verify Conjecture 1.1 (a) for group-type BVSs (see Corollary 4.17 and Conjecture 1.1(b) for Gaussian BVSs 
(see Proposition [3.6). We give a generalization of BVSs of group-type to quasiBVSs of group-type and prove our results in this more general setting.

The Gaussian braid representations here are (generalizations of) those considered in [16]. In loc. cit. these appear as the braiding for anyonic models generalizing Ising anyons and Majorana zero modes. The associated TQFT is the $S O(N)_{2}$ Chern-Simons theory, a fact that is established in 29. Similarly, the braid representations associated with group-type BVSs considered here are generalizations of those obtained from Dijkgraaf-Witten TQFTs ([7]).

\section{Preliminary Results}

Conjecture 1.1 should be compared with [28, Conjecture 2.7], where the predicted conclusion is that $\rho^{c}\left(\mathcal{B}_{n}\right)$ is finite modulo its center, assuming that $c$ has finite order. Our minor strengthening is due to the following:

Lemma 2.1. If c has finite order and $\rho^{c}\left(\mathcal{B}_{n}\right)$ is finite modulo its center $Z^{c}$ then $\rho^{c}\left(\mathcal{B}_{n}\right)$ is itself finite.

Proof. We decompose $\rho^{c}$ into irreducible representations $\rho_{i}$ and denote by $Z_{i}=\mathbb{C} I_{i}$ the center of $\rho_{i}\left(\mathcal{B}_{n}\right)$. As $c$ has finite order, the images of the generators $\sigma_{j}$ in $\rho_{i}\left(\mathcal{B}_{n}\right)$ also have finite order. Therefore, every element of $\rho_{i}\left(\mathcal{B}_{n}\right)$ has determinant a root of unity (of finite order). Now suppose $\beta \in Z^{c}$ with decomposition $\beta=\sum_{i} \lambda_{i} I_{i}$ as an element of $Z^{c}=\sum_{i} Z_{i}$. Now we see that the determinant of $\lambda_{i} I_{i}$ is a root of unity, and hence so is $\lambda_{i}$. Therefore $\beta$ has finite order. Thus the finitely generated abelian group $Z^{c}$ is finite, as each element has finite order. Thus $Z^{c}$ is finite and the result follows.

One large class of examples that obviously satisfy Conjecture 1.1(a) are the socalled locally monomial BVSs obtained as follows: Let $(S, X)$ be a set-theoretical solution (see [10]) to the braid equation, i.e., $S: X \times X \rightarrow X \times X$ satisfies the Yang-Baxter equation in $\operatorname{Aut}\left(X^{\times 3}\right)$. Then $S$ gives rise to a $\operatorname{BVS}(V, S)$ via linearization. Now choose a unitary diagonal matrix $D$ such that $(V, D S)$ is a BVS. Observe that with respect to the basis $X, D S$ is a monomial matrix so that $\rho^{D S}\left(\mathcal{B}_{n}\right)$ is isomorphic to a subgroup of the group $M_{|X|^{n}}(\mathbb{T})$ of $|X|^{n} \times|X|^{n}$ monomial matrices with entries on the unit circle $\mathbb{T}$. Now $M_{|X|^{n}}(\mathbb{T})$ is isomorphic

to $\mathfrak{S}_{|X|^{n}} \rtimes \mathbb{T}^{|X|^{n}}$ (a wreath product) which has the abelian group $\mathbb{T}^{|X|^{n}}$ as a finite index subgroup.

This example motivates the following:

Definition 2.2. - A locally monomial BVS consists of the data $(V, c, X$, $\left.\left(V_{x}\right)_{x \in X}\right)$, where $(V, c)$ is a BVS, $X$ is a finite set, and $\left.\left(V_{x}\right)_{x \in X}\right)$ is a family of one dimensional subspaces of $V$ indexed by $X$, such that $V=\bigoplus_{x \in X} V_{x}$ and $c$ permutes the spaces $V_{x} \otimes V_{y}$ for all $x, y \in X$. 
- A BVS $(V, c)$ is called locally monomializable if there exist a finite set $X$ and a decomposition $\left(V_{x}\right)_{x \in X}$, such that $\left(V, c, X,\left(V_{x}\right)_{x \in X}\right)$ is a locally monomial BVS.

- Two locally monomial BVSs $\left(V, c_{V}, X,\left(V_{x}\right)_{x \in X}\right)$ and $\left(W, c_{W}, Y,\left(W_{y}\right)_{y \in Y}\right)$ are monomial equivalent if there is an isomorphism $f: V \rightarrow W$ of BVSs such that $f\left(V_{x}\right)=W_{y}$ for all $x \in X$.

Remark 2.3. 1. The braiding of a locally monomial BVS induces a set-theoretical solution of the Yang-Baxter equation over $X$.

2. A BVS can have more than one inequivalent locally monomial structure.

3. If $(X, S)$ is a set-theoretical solution of the Yang-Baxter equation all possible locally monomial structures (up to diagonal isomorphism) are in oneto-one correspondence with the second Yang-Baxter cohomology group $H_{Y B}^{2}\left(X, \mathbb{C}^{*}\right)$, see [4].

4. Let $\left(V, c_{V}, X,\left(V_{x}\right)_{x \in X}\right)$ be a locally monomial BVS with $(X, S)$ the associated set-theoretical solution of the Yang-Baxter equation. If there is a graded basis $\left\{v_{x} \in V_{x}\right\}_{x \in X}$, such that the scalars of $c\left(v_{x} \otimes v_{y}\right)=$ $q_{x, y} v_{s_{1}(x, y)} \otimes v_{s_{2}(x, y)}$ are roots of the unity, then the image of the representation of $\mathcal{B}_{n}$ is a finite group.

\section{Gaussian Braided Vector Spaces}

In this section we construct unitary, finite order BVSs of dimension $m$ generalizing the "Gaussian" representations found in [15, 21, 20], in which the $m$ odd cases are found. We give full details as the case $m$ even is new, see also Remark 3.3 .

Let $m \in \mathbb{N}$ and define $q=\left\{\begin{array}{ll}e^{2 \pi i / m}, & m \text { odd } \\ e^{\pi i / m}, & m \text { even. }\end{array}\right.$ Define, as in [20], ES $(m, n-1)$ to be the algebra generated by $u_{1}, \ldots, u_{n-1}$ subject to: $u_{i}^{m}=1, u_{i} u_{i+1}=q^{2} u_{i+1} u_{i}$ and $u_{i} u_{j}=u_{j} u_{i}$ for $|i-j|>1$.

Proposition 3.1. The mapping $\varphi_{n}\left(\sigma_{i}\right)=\frac{1}{\sqrt{m}} \sum_{j=0}^{m-1} q^{j^{2}} u_{i}^{j}$ defines a representation of $\mathcal{B}_{n}$ into $E S(m, n-1)$ which becomes a $*$-representation upon setting $u_{i}^{*}=u_{i}^{-1}$.

We will need the following result, found in [21, Example 2.18 and Section 3.3]:

Lemma 3.2. Let $G$ be a cyclic quotient of $\mathbb{Z}$ (i.e., written additively) and $f: G \rightarrow$ $\mathbb{C}^{\times}$a function satisfying:

(a) $f(g)=f(-g)$

(b) $\frac{1}{|G|} \sum_{h \in G} \frac{f(h)}{f(g-h)}=\delta_{g, 0}$

(c) $\sqrt{|G|} \frac{f(x+y)}{f(x) f(y)}=\sum_{g \in G} \frac{f(g-y) f(g+x)}{f(g)}$.

Then $\varphi^{f}: \sigma_{i} \rightarrow \sum_{g \in G} f(g) u_{i}^{g}$ gives a homomorphism $\mathcal{B}_{n} \rightarrow E S(m, n-1)$. 
We now proceed to:

Proof of Proposition 3.1. Let $m$ be odd and $G=\mathbb{Z} / m \mathbb{Z}$. Define $f(a)=K q^{a^{2}}$ where $q=e^{2 \pi i / m}$ as above and $K$ is a constant to be determined. Clearly $(a)$ is satisfied while $(b)$ is easily verified:

$$
\sum_{h \in G} \frac{f(h)}{f(g-h)}=\sum_{h=0}^{m-1} q^{h^{2}-(g-h)^{2}}=q^{g^{2}} \sum_{h} e^{4 g h \pi i / m}=m \delta_{g, 0} .
$$

To verify $(c)$ we must show:

$$
\sqrt{m} \frac{f(x+y)}{f(x) f(y)}=\sqrt{m} q^{2 x y} / K=\sum_{g=0}^{m-1} \frac{f(g-y) f(g+x)}{f(g)}=q^{x^{2}+y^{2}} K \sum_{g=0}^{m-1} q^{g^{2}+2 g(x-y)} .
$$

Completing the square we see that it suffices to find a constant $K$ satisfying:

$$
\sqrt{m} / K^{2}=\sum_{g=0}^{m-1} q^{(g+(x-y))^{2}}=\sum_{g=0}^{m-1} q^{g^{2}}
$$

since $\{(g+(x-y)): 0 \leq g \leq m-1\}$ is a complete set of residues modulo $m$ (for any fixed $x, y)$. By Gauss' famous result we have $\sum_{g=0}^{m-1} q^{g^{2}}=\left\{\begin{array}{ll}\sqrt{m} & m=1(\bmod 4) \\ i \sqrt{m} & m=3(\bmod 4)\end{array}\right.$. Thus, $K^{2}=1$ or $K^{2}=-i$ will give solutions in these two cases. In order to get a *-representation of $\mathcal{B}_{n}$ in $E S(m, n-1)$ one should rescale by $1 / \sqrt{m}$.

Now let $m$ be even and $G=\mathbb{Z} / m \mathbb{Z}$. Recall that here $q=e^{\pi i / m}$. Define $f(a)=K q^{a^{2}}$ as above. Conditions $(a)$ and $(b)$ are verified in the same way as in the odd case, noting that $\sum_{h} e^{2 \pi g i / m}=m \delta_{g, 0}$. Condition $(c)$ reduces to verifying that $\sqrt{m} / K^{2}=\sum_{g=0}^{m-1} q^{(g+(x-y))^{2}}$ has a constant solution $K$. Since $q$ is a $2 m$ th root of unity, this is not a standard quadratic Gauss sum. However, $(g-k)^{2}=g^{2}$ $(\bmod 2 m)$ so that

$$
\sum_{g=0}^{m-1} e^{\frac{2 \pi i(g-k)^{2}}{2 m}}=\frac{1}{2} \sum_{g=0}^{2 m-1} e^{\frac{2 \pi i g^{2}}{2 m}}=\frac{(i+1) \sqrt{2 m}}{2}
$$

(see [5, Chapter 2]). Thus either choice for $K^{-2}=e^{\pi i / 4}=\frac{1+i}{\sqrt{2}}$ gives a solution.

Remark 3.3. - If we were to use $q=e^{\frac{2 \pi i}{m}}$ for $m$ even the operator $\sum_{j=0}^{m-1} q^{j^{2}} u_{i}^{j}$ would not be invertible.

- In [21] the formula for $f$ given as $K e^{\frac{\pi i a^{2}}{m}}$ with $K^{-2}=\sum_{g=0}^{m-1} e^{\frac{2 \pi g^{2} i}{m}}$ has typos, which are corrected in [15, 20]. 
3.1. Gaussian BVSs and Braid Group Images. To produce a Gaussian BVS it is enough to exhibit a vector space $V$ and an operator $U \in \operatorname{Aut}\left(V^{\otimes 2}\right)$ so that

$$
u_{i} \rightarrow U_{i}:=I_{V}^{\otimes i-1} \otimes U \otimes I_{V}^{\otimes n-i-1}
$$

extends to an algebra homomorphism $E S(m, n-1) \rightarrow \operatorname{End}\left(V^{\otimes n}\right)$, i.e. a localization of $E S(m, n-1)$. Recalling that $q=e^{2 \pi i / m}$ for $m$ odd and $q=e^{\pi i / m}$ for $m$ even, $\left\{\mathbf{e}_{i}: 0 \leq i \leq m-1\right\}$ be the standard basis for $V=\mathbb{C}^{m}$ and define a $U \in \operatorname{End}\left(V^{\otimes 2}\right)$ by

$$
U\left(\mathbf{e}_{i} \otimes \mathbf{e}_{j}\right)=q^{j-i} \mathbf{e}_{i+1} \otimes \mathbf{e}_{j+1},
$$

where $\mathbf{e}_{i+m}:=\mathbf{e}_{i}$.

Proposition 3.4. The map $u_{i} \rightarrow U_{i}:=I^{\otimes i-1} \otimes U \otimes I^{\otimes n-i-1} \in \operatorname{End}\left(V^{\otimes n}\right) d e-$ fines a *algebra homomorphism $\operatorname{ES}(m, n-1) \rightarrow \operatorname{End}\left(V^{\otimes n}\right)$. Moreover, $R:=$ $\frac{1}{\sqrt{m}} \sum_{j=0}^{m-1} q^{j^{2}} U^{j}$ is a unitary operator.

Proof. It is immediate that $U_{i}$ commutes with $U_{j}$ if $|i-j|>1$. Moreover,

$$
U^{m}\left(\mathbf{e}_{i} \otimes \mathbf{e}_{j}\right)=q^{(j-i)(m-j)} q^{(m-j+i)(j-i)} q^{(j-i) i}\left(\mathbf{e}_{i} \otimes \mathbf{e}_{j}\right)=\left(\mathbf{e}_{i} \otimes \mathbf{e}_{j}\right) .
$$

To check the remaining relation in $E S(m, n-1)$ it suffices to check the case $n=3$ with $U_{1}$ and $U_{2}$ :

$$
\begin{array}{r}
U_{1} U_{2}\left(\mathbf{e}_{i} \otimes \mathbf{e}_{j} \otimes \mathbf{e}_{k}\right)=q^{1+k-i} \mathbf{e}_{i+1} \otimes \mathbf{e}_{j+2} \otimes \mathbf{e}_{k+1}= \\
q^{2} q^{k-i-1} \mathbf{e}_{i+1} \otimes \mathbf{e}_{j+2} \otimes \mathbf{e}_{k+1}=q^{2} U_{2} U_{1}\left(\mathbf{e}_{i} \otimes \mathbf{e}_{j} \otimes \mathbf{e}_{k}\right) .
\end{array}
$$

Since $U^{-1}=U^{*}$ (conjugate-transpose) the homomorphism respects the $*$-structure on $E S(m, n-1)$ and hence $R$ is unitary.

We will call the pair $(V, R)$ above a Gaussian BVS.

Let $R_{i}(k):=\frac{1}{\sqrt{k}} \sum_{j=0}^{k-1} q^{j^{2}} u_{i}$ be the braid operators in $E S(k, n-1)$ where $q$ is a $k$-th (or $2 k$-th) root of unity for $k$ odd (resp. $k$ even).

Lemma 3.5. Suppose that we have prime factorization $m=p_{1}^{a_{1}} p_{2}^{a_{2}} \cdots p_{s}^{a_{s}}$. Then there exists Galois automorphisms $\tau_{h} \in \operatorname{Gal}\left(\mathbb{Q}_{p_{h}}^{a_{h}}\right)$ so that

$$
R_{i}(m)=\prod_{h=1}^{s} \tau_{h}\left(R_{i}\left(p_{h}^{a_{h}}\right)\right) .
$$

In particular, if $G_{h}$ is the image of $\mathcal{B}_{n}$ in $E S\left(p_{h}^{a_{h}}, n-1\right)$ then the image of $R_{i}(m)$ is a (diagonal) subgroup of $\prod_{h=1}^{s} \tau_{h}\left(G_{h}\right)$.

Proof. The key observation here is the following: if $x$ and $y$ are coprime then $u_{i}^{x}$ and $u_{j}^{y}$ commute for all $i$ and $j$, and $u_{i}^{x}$ generates an algebra of the form $\tau(E S(y, n-1))$ and vice versa. So in particular $E S(x y, n-1)$ factors as a direct product of algebras 
isomorphic to $E S(x, n-1)$ and $E S(y, n-1)$. The Chinese Remainder Theorem permits one to find automorphisms $\gamma_{x}$ and $\gamma_{y}$ so that $R_{i}(x y)=\gamma_{x}\left(R_{i}(x)\right) \gamma_{y}\left(R_{i}(y)\right)$.

We may now verify Conjecture 1.1(b) for the Gaussian BVS $(V, R)$. Setting $R_{i}=\rho^{R}\left(\sigma_{i}\right)$ as usual we have:

Proposition 3.6. The group $G_{n}$ generated by $R_{1}, \ldots, R_{n-1}$ is a finite group.

Proof. Firstly, since $G_{n-1} \subset G_{n}$, it is enough to consider $n$ odd. By an easy calculation we find

$$
\begin{aligned}
& R_{i} u_{i+1} R_{i}^{-1}=q u_{i}^{-1} u_{i+1} \\
& R_{i} u_{i-1} R_{i}^{-1}=q^{-1} u_{i-1} u_{i} .
\end{aligned}
$$

Therefore, the conjugation action of $G_{n}$ on the finite set $T_{n}:=\left\{q^{a_{0}} u_{1}^{a_{1}} \cdots u_{n-1}^{a_{n-1}}\right.$ : $\left.0 \leq a_{i} \leq 2 m-1\right\}$ gives a homomorphism $\Psi_{n}: G_{n} \rightarrow \operatorname{Sym}\left(T_{n}\right)$. The kernel of $\Psi_{n}$ is a subgroup of the center of $E S(m, n-1)$ which consists of scalar multiples of 1 (for $n$ odd). Since the eigenvalues of $R_{i}$ are roots of unity (of finite order) the determinant of any element of $\operatorname{ker}\left(\Psi_{n}\right)$ is also a finite order root of unity. Since it is a scalar matrix it must have finite order.

\section{QUASI-BRAIDED VECTOR SPACES OF GROUP-TYPE}

The following definition appears in [1].

Definition 4.1. A braided vector space $(V, c)$ is said to be of group-type if there exists a basis $\left\{x_{1}, \ldots, x_{n}\right\}$ for $V$ and $g_{i} \in G L(V)$ such that $c\left(x_{i} \otimes z\right)=g_{i}(z) \otimes x_{i}$ for all $z \in V$. We will call such a basis a braided basis for $c$.

In this section we will give an equivalent definition of group-type BVSs which can be extended to group-type quasi-BVSs. We will then verify Conjecture 1.1(a) for these classes of BVSs.

4.1. Twisted Yetter-Drinfeld modules over a group. Let $G$ be a group and $\omega \in Z^{3}(G, U(1))$ a 3-cocycle. Let us define $\gamma: G \times G \rightarrow \operatorname{Map}(G, U(1))$ by

$$
\gamma_{\sigma, \tau}(\rho):=\frac{\omega(\sigma, \tau, \rho) \omega\left(\sigma \tau \rho(\sigma \tau)^{-1}, \sigma, \tau\right)}{\omega\left(\sigma, \tau \rho \tau^{-1}, \tau\right)}
$$

and $\mu: G \rightarrow \operatorname{Map}(G \times G, U(1))$ by

$$
\mu_{\sigma}(\tau, \rho):=\frac{\omega\left(\sigma \tau \sigma^{-1}, \sigma, \rho\right)}{\omega\left(\sigma \tau \sigma^{-1}, \sigma \rho \sigma^{-1}, \sigma\right) \omega(\sigma, \tau, \rho)}
$$

for all $\sigma, \tau, \rho \in G$.

We denote by $y \mathcal{D}_{G}^{\omega}$ the category of Yetter-Drinfeld modules twisted by $\omega$, defined as follows: an object of $y \mathcal{D}_{G}^{\omega}$ is a vector space with a decomposition $V=\bigoplus_{\sigma \in G} V_{\sigma}$ 
and a compatible $\gamma$-twisted $G$-action, i.e., a map $G \times V \rightarrow V$, such that $\sigma V_{\tau} \subset$ $V_{\sigma \tau \sigma^{-1}}$ and

$$
(\sigma \tau) v_{\rho}=\gamma_{\sigma, \tau}(\rho) \sigma\left(\tau v_{\rho}\right)
$$

for all $v_{\rho} \in V_{\rho}$ and all $\sigma, \tau, \rho \in G$.

A morphism in $y \mathcal{D}_{G}^{\omega}$ is a linear map $f: V \rightarrow W$ such that $f\left(V_{\sigma}\right) \subset W_{\sigma}$ and $f(\sigma v)=\sigma f(v)$ for all $\sigma \in G, v \in V$.

The category of $\omega$-twisted Yetter-Drinfeld modules is braided with: the tensor product in $y \mathcal{D}_{G}^{\omega}$ is the tensor product of vector spaces with $G$-grading

$$
(V \otimes W)_{\sigma}=\bigoplus_{\tau \in G} V_{\sigma \tau^{-1}} \otimes W_{\tau},
$$

and twisted $G$-action $\sigma v_{\tau} \otimes w_{\rho}:=\mu_{\sigma}(\tau, \rho) \sigma v_{\tau} \otimes \sigma w_{\rho}$ for all $v_{\sigma} \in V_{\sigma}, w_{\tau} \in W_{\tau}$, $\sigma, \tau, \rho \in G$. The associator is given by

$$
\begin{aligned}
a_{V, W, Z}:( & (V \otimes W) \otimes Z \\
\left(v_{\sigma} \otimes w_{\tau}\right) \otimes z_{\rho} & \mapsto \omega(\sigma, \tau, \rho) v_{\sigma} \otimes\left(w_{\rho} \otimes z_{\rho}\right)
\end{aligned}
$$

and the braiding by $c_{V, W}\left(v_{\sigma} \otimes w_{\tau}\right)=\sigma w_{\tau} \otimes v_{\sigma}$.

When $\omega$ is trivial, $y \mathcal{D}_{G}^{\omega}$ is just the usual category of Yetter-Drinfeld modules over a group algebra (see [2]) and if $G$ is finite, $y \mathcal{D}_{G}^{\omega}$ is braided monoidally equivalent to the (modular) category $\operatorname{Rep}\left(D^{\omega} G\right)$ of representations of the twisted Drinfeld double $D^{\omega} G$, defined by Dijkgraaf, Pasquier and Roche [6].

The support of $V \in y \mathcal{D}_{G}^{\omega}$ is the set

$$
\operatorname{supp}(V)=\left\{g \in G: \operatorname{dim}\left(V_{g}\right) \neq 0\right\} .
$$

The following result show the connection between Yetter-Drinfeld-modules and BVSs of group-type.

Proposition 4.2. A BVS of group-type $(V, c)$ is the same as a finite-dimensional Yetter-Drinfeld module $V$ over a group $G$ such that the associated $G$-action is faithful and $G$ is generated by the support of $V$.

Proof. Let $(V, c)$ be a BVS of group-type with braided basis $\left\{x_{1}, \ldots, x_{n}\right\}$ and corresponding $\left\{g_{1}, \ldots, g_{n}\right\} \in G L(V)$. Define $G:=\left\langle g_{1}, \ldots, g_{n}\right\rangle \subset \operatorname{GL}(V)$ and $V_{g_{i}}:=\operatorname{span}\left\{x_{k} \mid g_{k}=g_{i}\right\}$. The braid equation on $c\left(x_{i} \otimes x_{j}\right)=g_{i}\left(x_{j}\right) \otimes x_{i}$ is equivalent to $g_{i} V_{g_{j}} \subset V_{g_{i} g_{j} g_{i}^{-1}}$. The converse is obvious.

For the rest of this section $(V, c)$ will denote a BVS of group-type, with $c\left(x_{i} \otimes\right.$ $\left.x_{j}\right)=g_{i}\left(x_{j}\right) \otimes x_{i}$ for some ordered braided basis $\left[x_{1}, \ldots, x_{n}\right]$ for $V$ and $g_{i} \in$ $G L(V)$. For notational convenience let $G=\left\langle g_{1}, \ldots, g_{n}\right\rangle$ be the subgroup of $G L(V)$ generated by the $g_{i}$.

Remark 4.3. We should emphasize that BVSs of group type are precisely all possible BVSs that can be obtained as finite dimensional Yetter-Drinfeld modules over an arbitrary group $G$. 
In [14, Definition 3.6], we defined the notion of quasi-braided vector spaces $(V, a, c)$ incorporating the possibility of non-trivial associativities through a family of isomorphisms $a$. Following the relation between BVSs of group-type and YetterDrinfeld modules (Proposition 4.2), we propose the definition of a quasi-BVS of group-type as follows:

Definition 4.4. A quasi-BVS of group-type is a finite-dimensional twisted Yetter-Drinfeld module over a group $G$ such that the associated twisted $G$-action is faithful and $G$ is generated by the support of $V$.

If $G$ is a finite group, the quasi-BVS of group type associated with a twisted $y \mathcal{D}$-module $V$ is the quasi-BVS of [14, Example 3.7]. In this case, we have the following:

Proposition 4.5. If $(V, c)$ is a quasi-BVS of group type and $|G|<\infty$ then $\left|\rho^{c}\left(\mathcal{B}_{n}\right)\right|$ is a finite group for all $n$.

Proof. One may identity $V$ with an object in $\operatorname{Rep}\left(D^{\omega} G\right)$ such that the braiding on $V^{\otimes 2}$ obtained from the universal $R$-matrix of $D G$ coincides with $c$ (see e.g. [1]). Now by [9, Theorem 4.2] the corresponding braid group representation has finite image.

4.2. Supporting subgroup structure. In light of Proposition 4.5, from here on we will assume that $G$ is an infinite group.

Let $V$ be a finite dimensional twisted $y \mathcal{D}$-module over an arbitrary group $G$. Since $V$ is finite $\operatorname{dimensional} \operatorname{supp}(V)$ is a finite set, and if $g \in \operatorname{supp}(V)$ the conjugacy class of $g$ is finite (since $\sigma V_{g} \subset V_{\sigma g \sigma^{-1}}$ ). If $F$ is the subgroup of $G$ generated by $\operatorname{supp}(V)$, then $V$ is a twisted $y \mathcal{D}$-module over $F$ and the representation of the braid group are exactly the same as the representation associated to the quasi-BVS $V$ as twisted $y \mathcal{D}$-module over $G$.

Therefore, as we are interested in the study of the quasi-BVSs associated with twisted $y \mathcal{D}_{G}^{\omega}$-modules, we can and will assume that $G$ is generated by a finite number of elements with finite conjugacy classes.

Lemma 4.6. Let $G$ be a group generated by a finite number elements each conjugacy class of which is finite. Then the center $Z(G)$ and centralizers $C_{G}(\sigma)$ have finite index for any $\sigma \in G$.

Proof. Let $\left\{g_{1}, \ldots, g_{n}\right\}$ be a set of generators such that each $g_{i}$ has a finite conjugacy class. Clearly

$$
z(G)=\bigcap_{i=1}^{n} C_{G}\left(g_{i}\right),
$$

i.e., an element of $G$ lies in the center if and only if it commutes with all the generators. Since for each $g_{i}$ we have $\left[G: C_{G}\left(g_{i}\right)\right]<\infty$ and $z(G)$ is an intersection of a finite number of subgroups of finite index, then $z(G)$ has also finite index. 
Now, since $z(G)$ has finite index and $z(G)=\bigcap_{\sigma \in G} C_{G}(\sigma), C_{G}(\sigma)$ has finite index for all $\sigma \in G$.

Corollary 4.7. If $(V, c)$ is a quasi-BVS of group-type with corresponding group $G:=\left\langle g_{1}, \ldots, g_{n}\right\rangle$, then $G / \mathcal{Z}(G)$ is a finite group.

Since $G$ is finitely generated then $z(G)$ is also finitely generated. Let $A$ the torsion-free subgroup of $z(G)$ and $H:=G / A$. Since $G / \mathcal{z}(G)$ and $z(G) / A$ are finite groups, $H$ is also finite.

The group $G$ is a central extension of $H$ by $A$. The central extensions of $H$ by $A$ are classified by elements of $H^{2}(H, A)$. If $A$ has rank $r$, i.e., $A \cong \oplus_{i=1}^{r} \mathbb{Z}$ then $H^{2}(H, A) \cong \oplus_{i=1}^{r} H^{2}(H, \mathbb{Z})$ and $H^{2}(H, \mathbb{Z}) \cong \operatorname{Hom}(H, U(1))$. Then if $H / H^{\prime} \cong$ $\oplus_{i=1}^{m} \mathbb{Z}_{n_{i}}$

$$
H^{2}(H, A) \cong \oplus_{i=1}^{m} A / n_{i} A .
$$

The 2-cocycles (hence the central extensions) can be constructed explicitly as follows: choose a 2-cocycle $f \in Z^{2}(U(1), \mathbb{Z})$ that represents the exact sequence

$$
\mathbb{Z} \rightarrow \mathbb{R} \rightarrow U(1)
$$

and define for $\chi \in \operatorname{Hom}\left(G, U(1)^{\times r}\right)$, the 2-cocycle $f_{\chi}: H \times H \rightarrow A:=\bigoplus_{i=1}^{r} \mathbb{Z}$, where

$$
f_{\chi}\left(h, h^{\prime}\right)=f^{\times r}\left(\chi(h), \chi\left(h^{\prime}\right)\right)
$$

for all $h, h^{\prime} \in H$. Note that by construction $f_{\chi}$ is symmetric. In fact, since $A$ is torsion-free for every finite group, $H^{2}(G, A)=\operatorname{Ext}\left(G / G^{\prime}, A\right)$.

4.3. Irreducible unitary twisted $y \mathcal{D}$-modules. In this subsection we will classify the finite dimensional irreducible twisted Yetter-Drinfeld modules.

4.3.1. Finite dimensional unitary projective representations of central extensions by finite groups. Let $G$ be a group and $\alpha \in Z^{2}(G, U(1))$ a 2-cocycle. Since every 2cocycle is cohomologous to a standard 2-cocycle, we will assume that $\alpha$ is standard, i.e., $\alpha\left(\sigma, \sigma^{-1}\right)=1$ for all $\sigma \in G$.

We define subgroups

$$
\begin{aligned}
\mathcal{Z}(G)(\alpha) & :=\{\sigma \in \mathcal{Z}(G): \alpha(\sigma, \tau)=\alpha(\tau, \sigma), \text { for all } \tau \in \mathcal{Z}(G)\}, \\
G(\alpha) & :=\{\sigma \in G: \alpha(\sigma, \tau)=\alpha(\tau, \sigma), \text { for all } \tau \in \mathcal{Z}(G)(\alpha)\} .
\end{aligned}
$$

It is not difficult to see that the groups $\mathcal{Z}(G)$ and $G(\alpha)$ only depend on the cohomology class of $\alpha$.

Example 4.8. Let $q$ be a primitive $n$-th root of unity. We can define a 2 -cocycle over $A=\mathbb{Z} \oplus \mathbb{Z}$, by $\alpha\left(a_{1} \oplus a_{2}, b_{1} \oplus b_{2}\right)=q^{a_{1} b_{2}}$, then $A(\alpha)=n \mathbb{Z} \oplus n \mathbb{Z}$.

Given a discrete group $G$ and a 2-cocycle $\alpha \in Z^{2}\left(G, \mathbb{C}^{*}\right)$, we will denote by $\mathbb{C}_{\alpha}[G]$ the group algebra twisted by $\alpha$, that is, $\mathbb{C}_{\alpha}[G]$ is the vector space with basis $\left\{u_{\sigma}\right\}_{\sigma \in G}$ and product $u_{\sigma} u_{\tau}=\alpha(\sigma, \tau) u_{\sigma \tau}$, for all $\sigma, \tau \in G$. The category 
of left $\mathbb{C}_{\alpha}[G]$-modules is canonically isomorphic to the category of $\alpha$-projective representation of $G$ and will be denoted by $\operatorname{Rep}\left(\mathbb{C}_{\alpha}[G]\right)$.

Let $G$ be any group, $N \unlhd G$ be a normal subgroup and $\pi: G \times G \rightarrow$ $G / N \times G / N$ the canonical projection. For any $\omega \in Z^{2}(G / N, U(1)), \operatorname{Inf}(\omega):=$ $\omega \circ \pi \in Z^{2}(G, U(1))$. The map Inf is called the inflation map and it defines a group homomorphism Inf: $H^{2}(G / N, U(1)) \rightarrow H^{2}(G, U(1))$. Note that the natural projection $\Pi: \mathbb{C}_{\operatorname{Inf}(\omega)}[G] \rightarrow \mathbb{C}_{\omega}[G / N]$ is an algebra epimorphism, so it defines a faithful functor

$$
\operatorname{Inf}(-):=\Pi^{*}(-): \operatorname{Rep}\left(\mathbb{C}_{\omega}[G / N]\right) \rightarrow \operatorname{Rep}\left(\mathbb{C}_{\operatorname{Inf}(\omega)}[G]\right) .
$$

The following theorem is a generalization of the main results of [3].

Theorem 4.9. Let $G$ be a finitely generated group such that $G / \mathcal{Z}(G)$ is finite and $\alpha \in Z^{2}(G, U(1))$ be a 2-cocycle. Then

(1) $G$ has a finite dimensional irreducible $\alpha$-projective $G$-representation if and only if $z(G)(\alpha)$ has finite index.

(2) If $\boldsymbol{Z}(G)(\alpha)$ has finite index, then there is a 2-cocycle

$$
\Omega \in Z^{2}(G(\alpha) / Z(G)(\alpha), U(1))
$$

such that $\left.\alpha\right|_{G(\alpha)}$ is cohomologous to $\operatorname{Inf}(\Omega)$.

(3) Assume that $\left.\alpha\right|_{G(\alpha)}=\operatorname{Inf}(\Omega)$ and that $\left\{U_{1}, \ldots, U_{l}\right\}$ is a representative set of the irreducible $\Omega$-representations of $G(\alpha) / Z(G)(\alpha)$ (since $G(\alpha) / Z(G)(\alpha)$ is finite, there are only finitely many isomorphism classes). Then all irreducible finite dimensional representations of $G$ are of the form

$$
\operatorname{Ind}_{G(\alpha)}^{G}\left(\theta \otimes \operatorname{Inf}\left(U_{i}\right)\right),
$$

where $\theta: G(\alpha) \rightarrow U(1)$ is a character.

(4) A pair of representations $\operatorname{Ind}_{G(\alpha)}^{G}\left(\theta \otimes \operatorname{Inf}\left(U_{i}\right)\right)$ and $\operatorname{Ind}_{G(\alpha)}^{G}\left(\theta^{\prime} \otimes \operatorname{Inf}\left(U_{j}\right)\right)$ are isomorphic if and only if $i=j$ and $\left.\theta\right|_{z(G)(\alpha)}=\theta_{z(G)(\alpha)}^{\prime}$.

Proof. Since $G$ is finitely generated, $z(G)$ is finitely generated, so every finite dimensional unitary projective representation of $z(G)$ is the direct sum of its irreducible subrepresentations. Let $V$ be a finite dimensional irreducible $\alpha$-representation of $G$. Then $\operatorname{Res}_{\mathcal{Z}(G)}^{G}(V)$ is a finite dimensional unitary $\alpha$-representation of $z(G)$, so by [3, Lemma 2], $[\mathcal{Z}(G): \mathcal{z}(G)(\alpha)]$ is finite and since $[G: \mathcal{Z}(G)]$ is finite, $[G: \mathcal{z}(G)(\alpha)]$ is finite.

We will apply Mackey's theory [26] to the finite index central subgroup z $(G)(\alpha)$ of $G$. Since $\alpha$ is symmetric on $z(G)(\alpha)$, its cohomological class is trivial and we may replace $\alpha$ by a 2 -cocycle such that $\left.\alpha\right|_{z(G)(\alpha)} \equiv 1$. Let $V$ be a unitary finite dimensional $\alpha$-projective representation of $G$. Since $\left.\alpha\right|_{z(G)(\alpha)} \equiv 1$, the restriction of $V$ to $z(G)(\alpha)$ decomposes as a direct sum of one dimensional linear representation of $z(G)$. It is easy to check that the little $\alpha$-group (i.e., stability group) of $G$ of any of these one dimensional $\mathcal{Z}(G)$ representations is equal to $G(\alpha)$ and only depends 
on $\alpha$. In particular $G(\alpha)$ is normal. By [26, Theorem 8.2] it follows that there exists an $\alpha$-projective irreducible unitary representation $W$ such that $V=\operatorname{Ind}_{G(\alpha)}^{G}(W)$. Since $G(\alpha)$ is normal, $V=\operatorname{Ind}_{G(\alpha)}^{G}(W)$ is isomorphic to $V^{\prime}=\operatorname{Ind}_{G(\alpha)}^{G}\left(W^{\prime}\right)$ if and only if $W$ is isomorphic to $W^{\prime}$ as $\alpha$-projective $G(\alpha)$-representations. Then the induction functor $\operatorname{Ind}_{G(\alpha)}^{G}(-)$ defines an equivalence between the category of finite dimensional unitary $\alpha$-projective representation of $G(\alpha)$ and the category of finite dimensional unitary $\alpha$-projective of $G$.

As in the proof of the main result of [3], we will apply Mackey's theory again, this time to the central subgroup $z(G)(\alpha)$ of $G(\alpha)$. Let $\chi: \mathcal{Z}(G)(\alpha) \rightarrow U(1)$ be a one dimensional representation of $z(G)$ and recall that the little $\alpha$-group of $G(\alpha)$ at $\chi$ is $G(\alpha)$ itself. By [26, Theorem 8.2] there exists a 2-cocycle $\Omega \in Z^{2}(G(\alpha) / Z(G)(\alpha))$ and a 1-cochain $\theta \in C^{1}(G(\alpha), U(1))$ such that

$$
\delta(\theta)=\alpha \operatorname{Inf}(\Omega)^{-1}
$$

Then the cohomology class of $\alpha$ and $\operatorname{Inf}(\Omega)$ are the same, so we can assume that $\alpha=\operatorname{Inf}(\Omega)$. Now, since $\alpha=\operatorname{Inf}(\Omega)$, equation (4.3) implies that $\theta$ is a character, so again by [26] (see also [22, Theorem 6.4.2]) the theorem follows.

Corollary 4.10. Let $G$ be a finitely generated group such that $G / \mathcal{Z}(G)$ is finite. Then

(1) There is a 2-cocycle $\Omega \in Z^{2}(G / Z(G), U(1))$ such that $\operatorname{Inf}(\Omega)$ has trivial cohomology and if $\left\{U_{1}, \ldots, U_{l}\right\}$ is a representative set of the irreducible $\Omega$-representations of $G / \mathcal{Z}(G)$, then all irreducible finite dimensional representations of $G$ are of the form

$$
\theta \otimes \operatorname{Inf}\left(U_{i}\right)
$$

where $\theta: G \rightarrow U(1)$ is a character.

(2) A pair of representations $\theta \otimes \operatorname{Inf}\left(U_{i}\right)$ and $\theta^{\prime} \otimes \operatorname{Inf}\left(U_{j}\right)$ are isomorphic if and only if $i=j$ and $\left.\theta\right|_{z(G)}=\theta_{\mathcal{Z}(G)}^{\prime}$.

Theorem 4.11. Let $G$ be a finitely generated group such that $G / Z(G)$ is $f$ nite and $\alpha \in Z^{2}(G, U(1))$ a 2-cocycle. Then every finite dimensional unitary $\alpha$-projective $G$-representation is a subrepresentation of a finite dimensional monomial $\alpha$-projective $G$-representation.

Proof. Since every finite dimensional unitary $\alpha$-projective $G$-representation is completely reducible, it is enough to proof the corollary for a finite dimensional irreducible unitary representation. By Theorem 4.9)(1)(2), we have that $G(\alpha) / Z(G)(\alpha)$ is a finite group and we can assume that $\left.\alpha\right|_{G(\alpha)}=\operatorname{Inf}(\Omega)$ for some

$$
\Omega \in Z^{2}(G(\alpha) / Z(G)(\alpha), U(1)) .
$$


By Theorem 4.9. (3), if $V$ is an irreducible finite dimensional unitary $\alpha$-representation, then $V \cong \operatorname{Ind}_{G(\alpha)}^{G}\left(\theta \otimes \operatorname{Inf}\left(U_{i}\right)\right)$, where $U_{i}$ is an irreducible $\Omega$-representation of $G(\alpha) / \mathcal{Z}(G)$. We can construct the finite dimensional monomial $\alpha$-projective representation $M(\theta):=\operatorname{Ind}_{G(\alpha)}^{G}\left(\theta \otimes \operatorname{Inf}\left(\mathbb{C}_{\Omega}[G(\alpha) / \mathcal{Z}(G)(\alpha)]\right)\right)$ and $V$ is a subrepresentation of $M(\theta)$.

\subsubsection{Classification of Twisted $y \mathcal{D}$-modules.}

Definition 4.12. $\quad$ Let $G$ be a group, $V$ be a vector space and $\pi: G \rightarrow$ $\mathrm{GL}(V)$ a map. A monomial structure on the map $\pi$ is a decomposition $V=\bigoplus_{x \in X} V_{x}$ into subspaces of dimension one, such that $\pi(\sigma)$ permutes the $V_{x}$ for all $x \in X$ and $\sigma \in G$.

- A monomial object in $y \mathcal{D}_{G}^{\omega}$ consist of a twisted $y \mathcal{D}$-module, with a monomial structure on the twisted $G$-action.

- A monomial projective representation is a projective representation $\pi: G \rightarrow \mathrm{GL}(V)$ with a monomial structure.

Fix $\chi \in H o m\left(H ; U(1)^{\times r}\right)$ and let $G:=A \rtimes_{\chi} H$ the associated central extension (see Subsection 4.2). For any subset $S \subset H$ we will denote the subset $\{(a, s): a \in$ $A, s \in S\}$ of $G$ by $A \rtimes_{\chi} S$.

Lemma 4.13. - For all $(a, \sigma) \in G, C_{G}((a, \sigma))=A \rtimes_{\chi} C_{H}(\sigma)$, in particular $z(G)=A \rtimes_{\chi} z(H)$.

- If $T$ is a representative set of conjugacy classes of $H$, then $A \rtimes_{\chi} T$ is a representative set of the conjugacy classes of $G$ and for all $(a, \sigma),[G$ : $\left.C_{G}((a, \sigma))\right]=\left[H: C_{H}(\sigma)\right]$.

Proof. It is easy to see that $(a, \sigma)$ commutes with $(b, \tau)$ if and only if $\sigma \tau=\tau \sigma$ and $f_{\chi}(\sigma, \tau)=f_{\chi}(\tau, \sigma)$, but by construction $f_{\chi}$ is symmetric so $(b, \tau) \in C_{G}((a, \sigma))$ if and only if $\tau \in C_{H}(\sigma)$. The second statement is a consequence of the first.

For each conjugacy class $R$ we denote by $y \mathcal{D}_{G}^{\omega}(R)$ the full abelian subcategory of $y \mathcal{D}_{G}^{\omega}$ consisting of the zero object and all twisted $y \mathcal{D}$-modules with support $R$. We will also denote by $(a, \sigma)^{G}$ the conjugacy class of $(a, \sigma)$ in $G$, that is, $(a, \sigma)^{G}=\left\{(a, \tau): \tau \in \sigma^{H}\right\}$.

Proposition 4.14. Let $T$ be a representative set of conjugacy classes of $H$.

(1) $y \mathcal{D}_{G}^{\omega}=\bigoplus_{(a, \sigma) \in A \rtimes_{\chi} T} y \mathcal{D}_{G}^{\omega}\left((a, \sigma)^{G}\right)$.

(2) The abelian categories $y \mathcal{D}_{G}^{\omega}\left((a, \sigma)^{G}\right)$ and $\operatorname{Rep}\left(\mathbb{C}_{\gamma_{-,-}(\sigma)}\left[A \rtimes_{\chi} C_{H}(\sigma)\right]\right)$ are equivalent.

(3) The finite dimensional irreducible unitary twisted $y \mathcal{D}$-modules are in oneto-one correspondence with triples $(\sigma, \theta, U)$, where $\sigma \in T, U$ is a finite dimensional unitary irreducible projective representation of

$$
A \rtimes_{\chi} C_{H}(\sigma)\left(\gamma_{-,-}(\sigma)\right) / z\left(A \rtimes_{\chi} C_{H}(\sigma)\right)\left(\gamma_{-,-}(\sigma)\right)
$$

and $\theta: A \rtimes_{\chi} C_{H}(\sigma)\left(\gamma_{-,-}(\sigma)\right) \rightarrow U(1)$ is a character. 
Proof. Statements (1) and (2) are simply restatements, for $G=A \rtimes_{\chi} H$, of the description in [6] of irreducible representations of $D^{\omega} G$ for $G$ finite. Statement (3) follows from Theorem 4.9.

Theorem 4.15. Every finite dimensional unitary twisted $y \mathcal{D}$-module over a group $G$ is a subobject and a quotient of a monomial twisted $y \mathcal{D}$-module over some group.

Proof. Since the category of finite dimensional unitary twisted $y \mathcal{D}$-modules over a group is semisimple, it is enough to prove that every unitary irreducible twisted $\mathrm{yD}$-module is a subobject and a quotient of a monomial twisted $\mathrm{y} \mathcal{D}$-module.

If $U$ is a monomial $\gamma_{-,-}(\sigma)$-projective representation of $A \rtimes_{\chi} C_{H}(\sigma)$, it follows by definition that the associated twisted $y \mathcal{D}$-module in Proposition 4.14 (see [6] for details of the construction) is monomial. If $V$ is a finite dimensional unitary irreducible twisted $y \mathcal{D}$-module, it is supported by only one conjugacy class. Let $U$ be the irreducible $\gamma_{-,-}(\sigma)$-projective representation of $A \rtimes_{\chi} C_{H}(\sigma)$ associated to $V$, it follows by Theorem 4.11 that there exists a monomial $\gamma_{-,-}(\sigma)$-projective representation of $A \rtimes_{\chi} C_{H}(\sigma)$ such that $U$ is a subobject and its associated twisted $y \mathcal{D}$-module is monomial and contains $V$.

Theorem 4.16. The image of the braid group representations associated to any finite dimensional unitary twisted $\mathrm{YD}$-module over a group $G$ is a quotient of a monomial representation of the braid group, defined via a monomial twisted $y \mathcal{D}$ module.

Proof. Let $V$ be a finite dimensional unitary twisted $y \mathcal{D}$-module. By Theorem 4.15 there exists a monomial twisted $y \mathcal{D}$-module $W$ such that $V$ is a quotient (or subobject) of $W$. By the functoriality of the braiding in $y \mathcal{D}_{G}^{\omega}$, the image of the representation of $\mathcal{B}_{n}$ is a quotient of the representation associated to $W$. Since $W$ is monomial, the associated representation of $\mathcal{B}_{n}$ is monomial.

We can now easily prove:

Corollary 4.17. The braid group image associated with any unitary quasi-BVS of group-type is virtually abelian.

Proof. A quasi-BVS of group-type is, by Definition 4.4, the same as a finite dimensional unitary twisted $y \mathcal{D}$-module over a group $G$ and the corresponding braid group images coincide. Since the image of any monomial representation of the braid group is virtually abelian the result follows.

\section{Discussion}

A classification of unitary BVSs in dimension higher than 2 seems computationally well out-of-reach. However, it is feasible that the monomial and Gaussian 
BVSs generate a large proportion of them (e.g. through quotients and subrepresentations). We are not aware of any BVSs that do not come from these two families.

We think the following questions are worth pursuing:

(1) Does Conjecture 1.1(a) imply (b)? That is, if $(V, c)$ is a BVS such that $c$ has finite order and the braid group image is virtually abelian does it follow that the image is actually finite?

(2) Does there exist a locally monomial BVS $(V, c)$ such that the braid group image is not finite modulo the center? By Lemma 2.1 $c$ must have infinite order. Moreover, there are monomial representations of $\mathcal{B}_{n}$ with infinite image modulo the center, the following gives an example:

$$
\sigma_{1} \rightarrow\left[\begin{array}{ccc}
0 & x & 0 \\
x & 0 & 0 \\
0 & 0 & \frac{1}{x^{2}}
\end{array}\right], \sigma_{2} \rightarrow\left[\begin{array}{ccc}
\frac{1}{x^{2}} & 0 & 0 \\
0 & 0 & x^{2} \\
0 & 1 & 0
\end{array}\right]
$$

(3) What are the images of the Gaussian representations? Goldschmidt and Jones [15] computed the braid group images in $E S(p, n-1)$ for $p$ an odd prime; they are (essentially) symplectic groups over the field with $p$ elements. For $p=2$ the images are extensions of extra-special 2 groups by symmetric groups ([12]). By Lemma 3.5, to understand the general Gaussian representation images it is enough to compute them for prime powers, i.e. in $E S\left(p^{k}, n-1\right)$.

(4) Turaev [31] introduced enhanced Yang-Baxter operators in order to produce link invariants from BVSs. What is the computational complexity of evaluating (approximately) the link invariants coming from locally monomial and Gaussian BVSs? For Gaussian BVSs at primes the invariants are computed in [15]. For related work, see [17.

\section{REFERENCES}

[1] N. Andruskiewitsch, H.-J. Schneider, Pointed Hopf algebras. New directions in Hopf algebras, 1-68, Math. Sci. Res. Inst. Publ., 43, Cambridge Univ. Press, Cambridge, 2002.

[2] N. Andruskiewitsch, M. Graña, Braided Hopf algebras over non-abelian finite groups. Bol. Acad. Nac. Cienc. (Córdoba), 63, 45-78 1999.

[3] N. B. Backhouse, On the form of finite dimensional projective representations of an infinite abelian group, Proc. Amer. Math. Soc. 41 (1973) no. 1, 294-298.

[4] J. S. Carter, M. Elhamdadi, M. Saito, Homology theory for the set-theoretic Yang-Baxter equation and knot invariants from generalizations of quandles, Fund. Math. 184 (2004) $31-54$.

[5] H. Davenport, Multiplicative number theory. Third edition. Graduate Texts in Mathematics, 74. Springer-Verlag, New York, 2000.

[6] R. Dijkgraaf, V. Pasquier, P. Roche, Quasi Hopf algebras, group cohomology and orbifold models. Recent advances in field theory (Annecy-le-Vieux, 1990). Nuclear Phys. B Proc. Suppl. 18B (1990), 6072 (1991). 
[7] R. Dijkgraaf, E. Witten, Topological gauge theories and group cohomology, Commun. Math. Phys. 129, (1990) 393-429.

[8] H. Dye, Unitary solutions to the Yang-Baxter equation in dimension four. Quantum information processing 2 (2002) nos. 1-2, 117-150 (2003).

[9] P. Etingof, E. C. Rowell, S. J. Witherspoon, Braid group representations from twisted quantum doubles of finite groups. Pacific J. Math. 234, no. 1 (2008), 33-41.

[10] P. Etingof, T. Schedler, A. Soloviev, Set-theoretical solutions to the quantum Yang-Baxter equation. Duke Math. J. 100 (1999), no. 2, 169209.

[11] J. M. Franko, Braid group representations arising from the Yang-Baxter equation. J. Knot Theory Ramifications 19 (2010), no. 4, 525538.

[12] J. Franko, E. C. Rowell, Z. Wang, Extraspecial 2-groups and images of braid group representations, J. Knot Theory Ramifications 15 (2006) no. 4, 1-15.

[13] M. H. Freedman, M. J. Larsen, Z. Wang, The two-eigenvalue problem and density of Jones representation of braid groups, Comm. Math. Phys. 228 (2002), no. 1, 177-199.

[14] C. Galindo, S.-M. Hong, E. C. Rowell, Generalized and quasi-localizations of braid group representations. Int. Math. Res. Not. IMRN 2013, no. 3, 693731.

[15] D. M. Goldschmidt, V. F. R. Jones, Metaplectic link invariants, Geom. Dedicata 131 (1989) no. 2, 165-191.

[16] M. B. Hastings, C. Nayak, Z. Wang, Metaplectic anyons, Majorana zero modes, and their computational power, Phys. Rev. B 87, (2013), 165421

[17] M. B. Hastings, C. Nayak, Z. Wang, On Metaplectic Modular Categories and their applications. arXiv:1303.1202.

[18] J. Hietarinta, All solutions to the constant quantum Yang-Baxter equation in two dimensions. Phys. Lett. A, 165 (1992), 2452-52.

[19] V. F. R. Jones, Braid groups, Hecke algebras and type $\mathrm{II}_{1}$ factors, Geometric methods in operator algebras (Kyoto, 1983), 242-273, Pitman Res. Notes Math. Ser. 123, Longman Sci. Tech., Harlow, 1986.

[20] V. F. R. Jones, On a certain value of the Kauffman polynomial. Comm. Math. Phys. 125 (1989), no. 3, 459467.

[21] V. F. R. Jones, On knot invariants related to some statistical mechanical models, Pacific J. Math. 137 (1989), no. 2, 311-334.

[22] G. Karpilovsky. Projective Representation of Finite Groups, volume 94. Pure and Applied Mathematics, Marcel Dekker, New York-Basel, 1985.

[23] L. Kauffman, S. Lomonaco Jr. Quantum entanglement and topological entanglement. New J. Phys. 4 (2002), 73.173.18.

[24] M. J. Larsen and E. C. Rowell, Unitary braid group representations with finite image. Algebr. Geom. Topol. 8 (2008), no. 4, 2063-2079.

[25] M. J. Larsen, E. C. Rowell, Z. Wang, The $N$-eigenvalue problem and two applications, Int. Math. Res. Not. 2005, no. 64, 3987-4018.

[26] G. W. Mackey, Unitary representations of group extensions. I, Acta. Math. 99 (1958), 265-311.

[27] D. Naidu and E. C. Rowell, A finiteness property for braided fusion categories, to appear in Algebr. Representation Theory. arXiv:0903.4157.

[28] E. C. Rowell, Z. Wang, Localization of unitary braid group representations, Comm. Math. Phys. 311 (2012) no. 3,343-389.

[29] E. C. Rowell, H. Wenzl, $S O(N)_{2}$ Braid group representations are Gaussian, in preparation. 
[30] E. C. Rowell, Braid representations from quantum groups of exceptional Lie type, Rev. Un. Mat. Argentina 51 (2010), no. 1, 165-175.

[31] V. G. Turaev, The Yang-Baxter equation and invariants of links. Invent. Math. 92 (1988), no. 3, 527-553.

[32] H. Wenzl, $C^{*}$ tensor categories from quantum groups. J. Amer. Math. Soc. 11 (1998) no. $2,261-282$.

Departamento de Matemáticas, Universidad de los Andes, Carrera 1 N. $18 \mathrm{~A}$ 10, Bogotá, Colombia

E-mail address: cn.galindo1116@uniandes.edu.co

Department of Mathematics, Texas A\&M University, College Station, TX 778433368

E-mail address: rowell@math.tamu.edu 